\title{
JUURNAL.RU
}

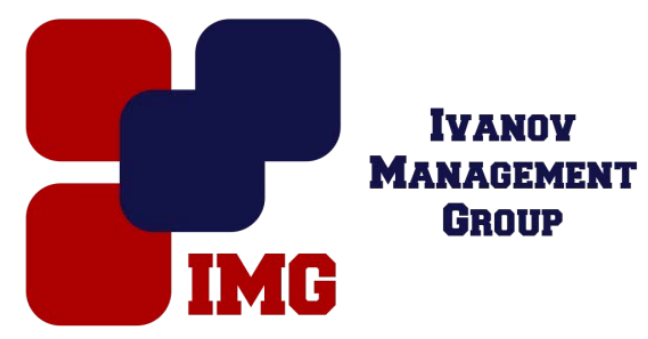

Карпенко М.В.

Московский Технологический Университет Москва, Россия

doi: 10.18411/lj-25-12-2016-1-02

idsp 000001:lj-25-12-2016-1-02

\section{Влияние функциональных возможностей проектируемой сети на ее конкурентоспособность}

При проектировании сенсорных сетей с заданной функциональностью обычно приходится формировать некоторый набор вариантов, каждый из которых удовлетворяет конкретным функциональным, эксплуатационным и стоимостным требованиям. Такой набор принято называть множеством допустимых проектных решений.

Формирование множества допустимых проектных решений оказывается довольно сложной задачей по двум причинам.

Во-первых, не всегда удается априори точно определить функциональные требования к разработанной системе к моменту ее изготовления. Во-вторых, предъявляемые функциональные требования могут меняться в зависимости от технической и рыночной конъюнктуры.

Вариативность предъявляемых к разрабатываемой системе требований определяет необходимое свойство множества допустимых проектных решений возможность быстрого и достаточно легкого перехода от одного элемента множества к другому.

Для успешного завершения этапа формирования множества допустимых вариантов разработки обычно используется механизм ранжирования вариантов с использованием состоятельной матрицы попарных сравнений, формируемой из экспертных данных.

Для успешного принятия решения о наилучшей реализации разрабатываемого варианта необходимо формировать множество наилучших вариантов (множество Парето), из которых эксперт уже выбирает наилучший вариант. Для нормальной работы эксперта на этом этапе необходимо, чтобы в сформированное множество входило немного вариантов (не более 2-3), характеристики которых достаточно наглядно различаются.

Для формирования такого множества наилучших вариантов удобно использовать интерактивный механизм, позволяющий эксперту корректировать векторный критерий отбора в процессе формирования множества. 
Множество допустимых вариантов разработки определяется тем, насколько полно эти варианты покрывают имеющийся спрос на функциональность сенсорной сети.

Сенсорные локальные сети - являются наиболее ярким представителем развития современных технологий в области проектирования электронных, сетевых и организационных систем. В настоящее время появилась возможность создавать недорогие маломощные и многофункциональные моты (узлы), которые «общаются» непосредственно друг с другом [1]. Сенсорные сети основаны на совместной работе большого числа крошечных узлов, которые состоят из модулей сбора и обработки данных и модуля связи. Такие сети имеет значительные преимущества перед набором традиционных датчиков. [1]:

Традиционные датчики характеризуются двумя важными особенностями

- Датчики могут быть расположены далеко от наблюдаемого явления, что требует много датчиков и использование достаточно сложных методов для фильтрации полезного сигнала из шума.

- Можно тщательно подобрать расположение некоторого количества датчиков, выполняющих сбор данных. Они будут передавать собранные данные в узлы, где и будет выполняются сбор и обработка данных.

Сенсорная сеть датчиков состоит из очень большого числа узлов (мотов), которые могут быть расположены близко к наблюдаемому явлению или объекту. Большое количество мотов не требует тщательного подбора мест их расположения. Это, с одной стороны, позволяет случайным образом располагать их в любых труднодоступных местах или использовать их для операций требующих быстрого реагирования. С другой стороны, сетевые протоколы и алгоритмы работы мотов должны быть достаточно сложными, чтобы обладать возможностью самоорганизации. Уникальной особенностью именно сенсорных сетей является возможность совместной работы отдельных узлов. Моты - это не только датчики, они оснащены микроконтроллером. Поэтому они могут не только передавать «сырые» данные, но и попутно обрабатывать их, выполняя простые вычисления и передавая далее только необходимые и/или частично обработанные данные. Указанные особенности обусловливают широкий спектр применения сенсорных сетей, и особенно явно проявляются их преимущества в рамках систем управления зданием (умным домом). Умный дом до предела насыщен всевозможными исполнительными механизмами, системами управления различного уровня - от примитивно-локальных, до общей системы управления домом. Структура взаимодействия системы управления умным домом обычно необычайно сложна - связи в этой структуре могут располагаться различным, иногда очень причудливым, образом. Именно в такой структурно и идеологически сложной системе управления и могут проявиться свойства сенсорных сетей датчиков, которые проще всего охарактеризовать словом «универсальность». Сочетание особенностей системы управления умным домом и сенсорных сетей датчиков определяет и особенности структурного проектирования таких систем с такими датчиками.

Рассмотрим основные различия сенсорных и одноранговых сетей [1]: 
- Количество узлов сенсорной сети может быть на несколько порядков выше, чем узлов в одноранговой сети.

- Узлы плотно расположены.

- Узлы подвержены сбоям.

- Топология сенсорных сетей может часто и хаотически изменяться

- Узлы в основном используют широковещательные сообщения, в то время как большинство одноранговых сетей основаны на связи "точка-точка".

- Узлы ограничены в питании, вычислительных мощностях, и памяти.

- Каждый из узлов трудно обеспечить уникальным идентификационным номером из-за большого их количества, а также из-за потенциальной недолговечности каждого узла.

К уникальным особенностям «умных датчиков»-узлов относится тот факт, что могут быть интегрированы в любые бытовые приборы, например в пылесосы, микроволновые печи, холодильники видеоплейеры. Они могут взаимодействовать друг с другом и с внешней сетью через Интернет или спутник. Это позволит конечным пользователям легко управлять устройствами дома как локально, так и удаленно.

Предлагается для оценки конкурентоспособности разработки определяется множество, состоящее из конечного числа допустимых вариантов, представляющих допустимый баланс уровня функциональной обеспеченности всех возможных областей применения устройства и затрат противостоящих конкурентов Х и Ү. Элементы $\omega_{j}(j=1, \ldots p)$ множества $\Omega$, представляющие устойчивые допустимые варианты, являются векторами [2]:

$$
\omega_{j}=\left(\alpha_{1 j}, \alpha_{2 j}, \ldots \alpha_{n j} ; \beta_{j 1}, \beta_{2 j}, \ldots \beta_{n j}\right),
$$

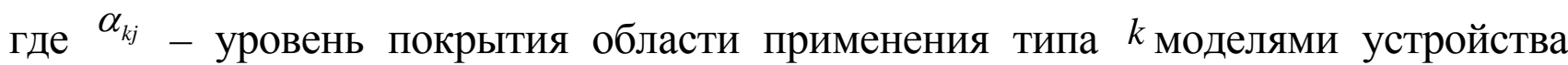
конкурента $\mathrm{X}$ в состоянии $j$, а $\beta_{k j}$ - аналогичный параметр для конкурента $\mathrm{Y}$

Здесь следует отметить, что степень привлекательности варианта разработки зависит не только от функциональности сенсорной сети, но также и от изменения спроса на те или иные функции. Кроме того, необходимо иметь в виду, что такой параметр разрабатываемой системы, как стоимость (себестоимость), зависит не только от конструктивных особенностей компонентов сети и их элементной базы, но и от изменения рыночных цен на комплектующие и на услуги третьих фирм (изготовление печатных плат, монтаж и т.д.). Влияние этих факторов можно проиллюстрировать, например, изменением цен на аналого-цифровые преобразователи (АЦП). Так стоимость 12-разрядного АЦП уменьшилась за 5 лет в десять раз, а его быстродействие увеличилось в несколько раз. Все это позволяет говорить о том, что оценка степени привлекательности вариантов разрабатываемой сенсорной сети не может оцениваться один раз в начале проектирования, эту оценку необходимо повторять многократно в течение всего времени жизни системы. 


\section{Литература}

1. [Электронный ресурс] - $]$ Режим доступа: http://wiki.amperka.ru/беспроводная-связь:настройка-хbee-series-2

2. Иваненко В.А, Зеленин А.Н. Информационные аспекты при разработке сенсорных сетей (часть 1). [Электронный ресурс[ - Режим доступа: http://cyberleninka.ru/article/n/informatsionnye-aspekty-pri-razrabotkesensornyh-setey-chast-1.

3. Кисляков М. А., Мосин С. Г., Савенкова В. В. Проектирование беспроводных сенсорных сетей. Известия высших учебных заведений «Приборостроение», № 8, 2012. - С. 15-18. 